Published in final edited form as:

Gerontechnology. 2016 ; 14(3): 169-176.

\title{
Subjective cognitive complaints and objective memory performance influence prompt preference for instrumental activities of daily living
}

\author{
Emily J. Van Etten ${ }^{\mathrm{a}}$, Alyssa Weakley, $\mathrm{MS}^{\mathrm{b}}$, Maureen Schmitter-Edgecombe, $\mathrm{PhD}^{\mathrm{b}}$, and \\ Diane Cook, PhDc \\ aSan Diego State University, Department of Psychology, San Diego, California, USA \\ bWashington State University, Department of Psychology, Pullman, Washington, USA \\ 'Washington State University, School of Engineering and Computer Science, Pullman, \\ Washington, USA
}

\section{Abstract}

Introduction-Declines in memory and executive functioning often lead to difficulties completing instrumental activities of daily living (IADLs). Prompting technologies have the potential to help promote aging in place by providing support for the initiation and accurate completion of IADLs. In this study, we evaluate preferences of older adults for different levels of prompting support based on subjective and objective measures of cognitive functioning.

Method-Participants were 170 community-dwelling older adults split into two cognitive complaint groups: cognitive complaints and few cognitive complaints. After completing six IADL tasks (e.g., organize a pillbox, cook), each participant was asked to make a specific error (e.g., leave stove on) on three of the tasks. They were then prompted to correct the error with one of three different prompt modes: verbal indirect, verbal direct, multimodal verbal direct and video.

Results-The cognitive complaints group reported greater preference for the multimodal prompt compared to the few cognitive complaints group. The indirect prompt was the least preferred by both groups. Furthermore, participants who recalled less on objective memory measures preferred more support in terms of prompt mode. Executive functioning did not appear to be related to prompt preference.

Conclusion-Level of subjective cognitive complaints and objective memory performance may influence amount of support preferred in a prompt.

\section{Keywords}

older adult; aging; assistive technology; prompting; cognitive impairment

As people age, declines in memory and executive functioning often lead to difficulties completing instrumental activities of daily living ${ }^{1}$ (IADLs), including cooking and managing finances ${ }^{2}$. Given that the U.S. older adult population is projected to double by $2050^{3}$, the number of adults requiring assistance with IADLs is also anticipated to increase significantly. Intact IADLs enable older adults to age independently in their own living spaces $^{4}$, which is a goal of significant importance to many older adults ${ }^{5,6}$. Appropriate and 
acceptable technology-based interventions may provide a means by which older adults can age successfully in their own homes even in the face of functional declines.

Compared to nursing homes or other external facilities, research shows aging in place is beneficial to the health and well-being of older adults ${ }^{7-9}$ and reduces personal and public expenditures for health care and services ${ }^{6}$. Prompting technologies have the potential to help promote aging in place by providing support for the initiation and accurate completion of everyday activities of daily living, thereby maintaining safe and healthy independent living ${ }^{10-14}$. In this study, we evaluate preferences for different levels of prompting support (i.e., indirect, direct, multimodal) using subjective and objective measures.

Prompting technologies are devices that deliver verbal and/or visual cues to a user in order to help the user initiate, correct, or complete activities independently ${ }^{15}$. They can range from simple reminders delivered automatically at regular intervals (e.g., alarm set on phone), to more complex alerts involving machine learning algorithm systems that provide contextaware prompting ${ }^{14}$ (e.g., reminder to take pills after breakfast is complete). Although simple time-based prompting systems are useful ${ }^{11}$, these systems may deliver prompts when the user is involved in other activities, thereby limiting the ability of the user to initiate or carry out the prompted task at that given time, reducing the likelihood of task completion ${ }^{16}$. More complex machine learning algorithm systems attempt to adjust for this by using sensors and other technologies to learn and track an individual's behavior ${ }^{10,12,13}$. These systems can prompt users at appropriate times (e.g., when they are not engaged in other activities) and when necessary ${ }^{17-19}$ (e.g., when user leaves the stove on). For example, Pollack et al., developed a machine-learning prompting system, called Autominder ${ }^{19}$. The device will schedule and remind users to take their medication, but also take into account their preprogrammed daily schedule, such as their favorite television show, in order to not send reminders that conflict with another task a user is engaging in.

Multiple studies have found prompting devices can help individuals maintain performance of everyday tasks such as taking medications and hand washing $5,10,20$. Prompts can be delivered by different modes, including: $\operatorname{text}^{21}$, audio ${ }^{22}$, video ${ }^{23}$, or a combination of these methods ${ }^{14,24}$. For example, Wade and Troy had five individuals with traumatic brain injury pick specific IADLs as targets (e.g., making lunch), and then personalized audio prompts were delivered through a mobile phone to improve performance completing the IADLs ${ }^{25}$. Participants and their caregivers constructed the wording of prompts and picked their target IADLs. After 12 weeks, caregivers reported that each participant significantly improved performance on their target IADLs and benefitted from their specific prompt. Thus, different individuals may require different types of prompts. While different types of prompts have been shown to help individuals maintain performance on IADLs, there is little known about what type of prompt mode is preferred.

Without assistive technologies, a family member or friend often needs to provide cues or prompts to help an individual initiate or complete IADLs. The level of assistance required is often perceived as burdensome, leading to significant caregiver stress, burnout, and depression ${ }^{26}$. In an attempt to reduce caregiver support required, researchers have investigated the relationship between prompt level and cognition, using graded cue hierarchy 
systems ${ }^{16,27}$. Graded cue hierarchy systems start with prompts that provide less assistance and then move on to more directive prompts if needed. With these systems, the user only receives the assistance required, thereby, promoting independence.

Seelye and colleagues incorporated a graded cue hierarchy into technology-based prompts to assist with IADL completion (e.g., changing light bulb, cooking oatmeal) ${ }^{16}$. For example, when individuals with multi- or single-domain MCI (Mild Cognitive Impairment) and healthy older adults experienced difficulty with IADL completion, they received a preprogrammed prompt initiated by the experimenter through a laptop computer system. The prompting hierarchy began with a verbal indirect prompt that oriented the individual back to the task (e.g., "the oatmeal may burn if the stove is left on"), followed by a verbal direct prompt (e.g., "you can turn the stove off now"), and then a multimodal prompt (e.g., a video of a person appeared on computer screen turning the knob on the stove to the off position, along with a verbal direct prompt), if additional assistance was needed. Results revealed that multi-domain MCI participants nominally required significantly more prompts to accurately complete IADLs compared to single-domain MCI and healthy older adult participants.

However, there was no significant difference in the average level of prompt needed, with all participants responding equally well to the lowest level of prompting available: indirect verbal. These findings suggest that an indirect prompt may be enough to orient individuals with MCI back to the task. Similarly, in a sample of individuals with Parkinson's disease without dementia and healthy controls, Foster found that only a low level of cueing was necessary to facilitate IADL completion on the Performance Assessment of Self-care Skills, which assesses IADL completion within a laboratory context ${ }^{27}$. Although these studies identified the lowest level of prompt assistance required to support the accurate completion of IADLs, these studies did not examine prompt preferences.

In considering prompt preference, self-efficacy is generally described as an individual's belief in their capacity to execute behaviors and tasks ${ }^{28,29}$. Individuals with lower selfefficacy, compared to individuals with higher self-efficacy, are more likely to prefer to use strategies (e.g. lists, reminders) to complete everyday tasks ${ }^{30,31}$. Assistive technologies, such as prompting devices, can be considered helpful strategic devices that can assist individuals with completing IADLs accurately ${ }^{9}$. An individual who has low self-efficacy for their everyday cognitive functioning should be more likely to endorse requiring extra assistance ${ }^{32}$. Several studies have found a significant positive relationship between self-efficacy and objective measures of cognitive ability ${ }^{33,34}$. Thus, both subjective and objective measures may influence preference of prompt support level, such that individuals who have stronger cognitive abilities and/or perceive their cognitive abilities to be strong will report liking a less supportive prompt. Abandonment rate of assistive technology is high and older adults are more likely to utilize technologies they like and find beneficial ${ }^{14}$. Therefore, it is important to understand the preferences of older adults, with and without cognitive impairment, to establish and preserve use over time. The purpose of the current study is to evaluate preferences for different prompt modes among older adults with and without cognitive difficulties. We were especially interested in whether perceived and objective level of cognitive ability influences preference for type of prompt. The current study uses the same prompt modes as Seelye and colleagues (i.e., verbal indirect, verbal direct, and verbal direct/video multimodal prompts) to evaluate prompt mode preference ${ }^{16}$. In accordance with 
self-efficacy theory, we hypothesized that participants will show preference for higher levels of prompt support as their self-reported, or perceived, cognitive functioning declines. Furthermore, we hypothesized that participants who perform more poorly on objective cognitive measures will also show preference for a greater supportive prompt mode. Since executive functioning measures and memory measures have been found to significantly predict performance on IADLs ${ }^{1}$, the current study focuses on these cognitive factors and their relationship with prompt mode preferences.

\section{Method}

\section{Participants}

Participants were 170 community-dwelling older adults. Participants were recruited through health and wellness fairs, local referrals, advertisements, and from past laboratory studies. To exclude older adult participants who were likely to meet criteria for dementia, participants were first screened over the phone with a medical interview and the 'Telephone Interview for Cognitive Status ${ }^{35}$. Those who passed the initial screening were invited to complete a battery of standardized and experimental neuropsychological tests and completed IADL tasks in a campus apartment converted into a research testbed and outfitted with smart technology (e.g., infrared motion detectors). Participants were compensated with a report documenting their performance on the neuropsychological tests, parking, and travel. This protocol was reviewed and approved by the Institutional Review Board at Washington State University.

The majority of participants recruited were healthy older adults $(n=116)$. The sample also included fifty-four individuals with various medical conditions $(n=54)$ that did not meet criteria for dementia according to criteria outlined by the National Institute of Neurological and Communicative Disorders and Stroke and the Alzheimer's disease and Related Disorders Association ${ }^{36}$. See Table 1 for a breakdown of medical diagnosis and how many participants fell within each diagnostic category. Classification as MCI was established by consensus of two experienced neuropsychologist using established criteria ${ }^{37}$, Parkinson's disease by a board certified neurologist with specialization in movement disorders, and stroke, TBI (Traumatic Brain Injury) and other medical conditions (e.g., cancer) through participant self-report. In the present study, we chose not to focus on particular medical diagnoses of participants; rather, we opted to focus on subjective and objective measures of cognitive and functional difficulties.

\section{Procedure}

Participants were first given a 3-hour battery of neuropsychological tests. One week later, participants completed six different IADL tasks in a campus apartment testbed. These tasks included: organizing a pillbox, sweeping \& dusting, using the microwave in a cooking task, washing a countertop, watering plants, and washing their hands. After participants experienced each task they were requested to complete three of the six tasks again, but this time they were asked to make a specific error (e.g., leave stove on when cooking, leave water on when washing hands). After participants completed the requested error, experimenters watching live video feed in a control room upstairs intervened and delivered a 
prerecorded prompt through either a tablet or smart phone that was placed on participants' non-dominant forearm. All participants experienced each of the three prompt modes (i.e., indirect, direct, and multimodal). The activities prompted and the prompt modes were randomized and counterbalanced to control for activity and order effects. The indirect prompt was a verbal prompt that oriented the participant back on task, but did not tell them what their mistake was (e.g. "the oatmeal may burn if the stove is left on"). The direct prompt was a verbal prompt that told the participants exactly what to do (e.g. "you can turn the stove off now"). For the multimodal prompt, the device (tablet or phone) beeped first to orient the participant to the device, then the device showed a video of what needed to be done, along with providing a verbal direct prompt. After participants experienced each prompt mode they were asked to indicate which prompt mode they liked best.

\section{Measures}

The Patient Reported Outcomes Measurement Information System ${ }^{38}$ Applied CognitionAbilities Short Form was used to measure subjective cognitive complaints. Participants used a 5-point Likert scale ( $1=$ not at all; $5=$ very much) to rate their experienced difficulties during the past seven days on eight questions pertaining to different aspects of cognitive functioning, including memory and executive functioning. Participant scores were summed across questions to create an aggregated total score for each participant.

Derived immediate and long delay story recall scores from the Memory Assessment Scale $(\mathrm{MAS})^{39}$ were used to provide information about objective memory abilities. The MAS Story Memory subtest requires participants to learn and recall a short story. The full original story was determined to have 18 'units' of information that a person could freely recall (e.g., ' 3 men', 'Volkswagen', '2:30'). The total numbers of 'units' recalled both immediately and after a 30-minute delay were used as measures of immediate and delayed memory.

To provide information about participants' executive functioning abilities, the switching scores from the Verbal and Design Fluency subtests of the Delis-Kaplan Executive Function System ${ }^{40}$ were used for analyses. The Verbal Fluency Category Switching task requires participants to switch back and forth between naming a fruit and then a piece of furniture. The total number of times participants were able to correctly switch between fruits and furniture was used for analyses. The Design Fluency Switching task requires participants to connect dots using 4 straight lines, alternating each line between a filled dot and an empty dot. The total number of boxes that participants correctly connected was used for analyses.

\section{Analyses}

For perceived level of cognitive difficulties, a median split on the PROMIS Applied Cognition questionnaire ${ }^{38}$ was first conducted to classify participants into two cognitive complaints groups: cognitive complaints and few cognitive complaints. A chi-square test of independence was then used to determine whether the relationship between preferred prompt and classification of cognitive complaints and few cognitive complaints is independent or if the variables are significantly associated. For objective cognitive abilities, Spearman correlations were used to examine the relationship between objective measures of memory and executive functioning and prompt preferred. A hierarchical regression was also 
performed to determine whether memory and executive functioning measures would predict prompt preference, over and above demographic variables (age, education and gender).

\section{Results}

\section{Subjective cognitive complaints}

To create two groups that differed in self-report of cognitive difficulties, participant scores on the PROMIS Applied Cognition questionnaire were first split down the median $(M d n=31)$. Participants reporting scores of 32 or higher were classified as having 'cognitive complaints', consistent with the fact that the scores of participants in this group fell 1.5 standard deviations or more above the mean of the normative population. Participants who reported scores of 31 or below were classified as having 'few cognitive complaints'. As seen in Table 2, independent t-tests revealed the self-reported cognitive complaints groups did not differ in mean age or education.

Compared to the few cognitive complaints group, participants in the cognitive complaints group not only self-reported more cognitive difficulties but also performed significantly more poorly on both the immediate and delayed prose memory measures and the DKEFS Verbal Fluency switching measure, with moderate and small Cohen's d effect sizes, respectively (Table 2). The two groups did not differ significantly on the DKEFS Design Fluency subtest switching measure.

A chi-square test of independence examining preferred prompt and level of self-reported cognitive complaints was significant, $\mathrm{X}^{2}(2, \mathrm{~N}=170)=7.569, \mathrm{p}=0.023$. The adjusted residuals method was used in order to evaluate individual cell contributions to the chi-square results ${ }^{41,42}$. A Bonferroni correction was used in order to maintain an appropriate Type I error rate ${ }^{41}$. There are 6 cells in the $2 \times 3$ contingency table; therefore the alpha was set at approximately $0.008(0.05 / 6)$, which corresponds to a critical value of $+/-2.6$. The findings indicated that the amount of participants who preferred the multimodal prompt was significantly larger in the cognitive complaints group (49.4\%) and significantly smaller in the few cognitive complaints group (29.4\%) than would be expected by chance (adjusted residual=2.7). As seen in Table 3, the few cognitive complaints group preferred the direct prompt $(44.7 \%)$ to the other two prompts. Additionally, for both groups, the least liked prompt was the indirect prompt.

\section{Objective measures}

To examine the relationship between objective measures of memory and executive functioning and prompt preferred, Spearman correlations were performed. Spearman correlations were selected because the data were not normally distributed and prompt preference was coded as a rank order: 1 indicating the indirect prompt, 2 indicating the direct prompt, and 3 indicating the multimodal prompt; therefore, the higher the number, the more support the prompt was thought to give. Also, since age, education, and gender were not associated with prompt preference (age: $\mathrm{r}_{\mathrm{s}}=-0.037, \mathrm{p}=0.634$; education: $\mathrm{r}_{\mathrm{s}}=0.002, \mathrm{p}=$ 0.983; gender: $r_{s}=0.005, p=0.946$ ), these variables were not controlled for in these analyses. The MAS immediate, $r_{s}=-0.200, p=0.010$, and MAS delayed, $r_{s}=-0.222, p=0.004$, prose 
recall measures were both negatively correlated with prompt preferred. This suggests that as participants recalled less on both memory measures, they preferred more support in terms of prompt mode. In contrast, neither the DKEFS verbal switching, $\mathrm{r}_{\mathrm{s}}=-0.058, \mathrm{p}=0.453$, or de sign switching, $\mathrm{r}_{\mathrm{s}}=-0.082, \mathrm{p}=0.290$, executive functioning measures were significantly correlated with prompt preference.

To determine whether the memory and executive functioning measures could predict prompt preference, a hierarchical regression was performed. Age, education, and gender were entered in Block 1, followed by memory and executive functioning measures in Block 2. The hierarchical regression revealed that the demographic variables, $\mathrm{F}(3,160)=0.076$, $\mathrm{p}=0.973$, did not explain significant variance in prompt mode preference, accounting for only .1\% of the variance. In contrast, the executive functioning and memory measures explained an additional $9.8 \%$ of the variance and the change in $\mathrm{R}^{2}$ was significant, $\mathrm{F}(4,156)=2.454, \mathrm{p}=0.020$. The only significant cognitive predictor of prompt preference was the delayed prose memory score, $\mathrm{B}=-0.251, \mathrm{t}=-2.143, \mathrm{p}=0.034$. Next, to determine whether the memory measures would account for significant variance over and above the executive functioning measures, a hierarchical regression was conducted with demographics entered in block 1 , followed by the executive functioning measures in block 2 and then the memory measures in block 3 . The regression analysis revealed that together the demographic and executive functioning measures only accounted for $2.6 \%$ of the variance, with the memory measures accounting for an additional significant $7.3 \%$ of the variance, $F(2,156)=2.454$, $\mathrm{p}=0.020$. The regression statistics are displayed in Table 4 .

\section{Discussion}

In this study, community-dwelling older adults performed IADLs in a naturalistic setting. While completing IADLs, participants performed scripted mistakes and were provided with different levels of prompt support by the experimenter through an electronic device to rectify the mistakes. Subjective self-report and objective neuropsychological measures were used to determine the relationship between memory and executive functioning and prompt support level preferences. Findings revealed that individuals who self-reported experiencing cognitive problems desired a prompt mode that provided more support (i.e., a direct verbal cue and video showing them exactly what to do) compared to individuals who self-reported experiencing less cognitive problems. The results further indicated that difficulties with memory, in comparison to executive functioning, were an important cognitive factor influencing the level of support a person desired.

According to self-efficacy theory literature ${ }^{28,29}$, individuals who have lower self-efficacy for their ability to complete a task accurately and independently are more likely to prefer and/or use external aids ${ }^{30,31}$. Consistent with this theory, we found that participants who selfreported cognitive problems were more likely to prefer a greater level of support (i.e., multimodal) then those who self-reported fewer cognitive complaints (i.e., verbal direct prompt). The lowest prompt support level, the indirect prompt, was the least preferred prompt level by both groups. 
Although previous studies ${ }^{16,27}$ found low levels of prompt assistance (e.g., indirect) aided in the accurate completion of IADLs, these studies did not evaluate whether participants liked the indirect prompt. This may indicate that although the indirect prompt is sufficient for allowing individuals to correctly complete tasks, a more directive prompt appears to be the more desired type of prompt. This could reflect the fact that the direct prompt reduces ambiguity and the need for an individual to infer what they should do. Such ambiguity may provoke anxiety in an individual. Further, individuals are more likely to use prompting devices if it improves their self-efficacy ${ }^{9}$. Therefore, individuals may feel the indirect prompt offers too little support and may not improve their own judgment of self-efficacy for accurately completing IADLs.

Both executive functioning and memory measures have been shown to significantly predict performance on IADLs ${ }^{14}$. Difficulties with memory and executive functioning could therefore impact a participant's choice of prompt mode to assist with IADL completion. However, we found that memory measures significantly correlated with preferred prompt support level, while executive functioning measures did not. As participants performed more poorly on memory measures, they indicated preferring more support in a prompt, while executive functioning measures appeared to not affect preferred prompt support level. Furthermore, a hierarchical regression revealed that memory measures significantly predicted type of prompt preferred, after controlling for demographics and executive functioning measures. These findings suggest that, compared to executive functioning, memory is an important cognitive factor influencing the level of support a person may prefer in a prompt mode. Individuals experiencing memory difficulties might be concerned that they may not correctly remember what they need to do, thus preferring higher levels of support in a prompt.

These findings also suggest that prompting systems may work best if flexibility is built into the system. As individuals experience greater levels of cognitive difficulties, our findings suggest that they may prefer to have prompts that supply a higher level of cognitive support. In addition, in both the cognitive complaints and few cognitive complaints groups, there were a considerable amount of participants that preferred each of the three prompt modes. This may indicate that there are significant individual differences in preference for prompt mode and building flexibility into a prompting system may increase user satisfaction as well as longevity.

Limitations of the present study include a sample of predominately highly educated, Caucasian participants living in the USA, which limits the generalizability of our findings. A less-educated sample may not react to different prompt support levels in the same ways as a more educated sample because they may be less comfortable with using technology. To address this, future studies should aim to examine preferences of prompt support level in more diverse samples. Additionally, although this study is set in a naturalistic apartment testbed, this may not be reflective of an individuals' everyday environment. For example, there were no interruptions, distractions, and participants did not have to switch between different tasks while completing these scripted IADLs. Altogether, these factors may cause the IADLs to not be as challenging as a normal home environment, which may cause participants to have altered reactions to different prompting support levels. Future studies 
should assess preferred level of prompt support while completing IADLs in the home environment.

Naturalistic assessment of older adults using assistive technologies is an innovative way to understand and improve independence in healthy aging. Assistive technologies, such as prompting devices, have the ability to help older adults stay in their homes for longer and age in place benefitting the health of older adults ${ }^{7-9}$, lessening personal and public expenditures ${ }^{6}$, and reducing the amount of caregiver burnout ${ }^{26}$. Prompting technologies can help older adults initiate and accurately complete IADLs with more independence ${ }^{10-14}$. Yet, there is a high rate of abandonment of assistive technology; and older adults are more likely to utilize technologies they like and find useful ${ }^{14}$. These data suggests that individuals reporting subjective cognitive complaints and experiencing objective memory difficulties may prefer greater levels of support in a prompt. This may be relevant information for future investigators and creators of prompting devices to recognize. Future research is needed to further understand factors that contribute to the prompt preferences of older adults to better preserve use of assistive technologies over time.

\section{Acknowledgments}

This study was partially supported by a grant from the National Institutes of Biomedical Imaging and Bioengineering (\#R01 EB009675) and a grant from the National Institute on Aging (\#R25 AG046114), which funded an undergraduate summer fellowship to study in the field of Gerontechnology at Washington State University for Emily van Etten. No conflicts of interest exist.

\section{References}

1. Tomaszewski FS, Cahn-Weiner DA, Harvey DJ, Reed BR, Mungas D, Kramer JH, Chui H. Longitudinal changes in memory and executive functioning are associated with longitudinal change in instrumental activities of daily living in older adults. The Clinical Neuropsychologist. 2009; 23(3):446-461. DOI: 10.1080/13854040802360558 [PubMed: 18821181]

2. Dodge HH, Kadowaki T, Hayakawa T, Yamakawa M, Sekikawa A, Ueshima H. Cognitive impairment as a strong predictor of incident disability in specific ADL-IADL tasks among community-dwelling elders: the Azuchi study. The Gerontologist. 2005; 45(2):222-230. DOI: 10.1093/geront/45.2.222 [PubMed: 15799987]

3. Vincent, GK., Velkoff, VA. The next four decades: The older population in the United States: 2010 to 2050. US Department of Commerce, Economics and Statistics Administration, US Census Bureau; 2010.

4. Diehl M, Marsiske M, Horgas AL, Rosenberg A, Saczynski JS, Willis SL. The revised observed tasks of daily living: A performance-based assessment of everyday problem solving in older adults. Journal of Applied Gerontology. 2005; 24(3):211-230. DOI: 10.1177/0733464804273772 [PubMed: 18160968]

5. Boll S, Heuten W, Meyer EM, Meis M. Development of a multimodal reminder system for older persons in their residential home. Informatics for Health and Social Care. 2010; 35(3/4):104-124. DOI: 10.3109/17538157.2010.528651 [PubMed: 21133767]

6. Satariano W, Scharlach A, Lindeman D. Aging, place, and technology: Toward improving access and wellness in older populations. Journal of Aging and Health. 2014; 26(8):1373-1389. DOI: 10.1177/0898264314543470 [PubMed: 25502245]

7. Bryant LL, Corbett KK, Kutner JS. In their own words: a model of healthy aging. Social Science \& Medicine. 2001; 53(7):927-941. DOI: 10.1016/S0277-9536(00)00392-0 [PubMed: 11522138]

8. Cutchin MP. The process of mediated aging-in-place: A theoretically and empirically based model. Social Science \& Medicine. 2003; 57(6):1077-1090. DOI: 10.1016/S0277-9536(02)00486-0 [PubMed: 12878107] 
9. Intille SS. A new research challenge: persuasive technology to motivate healthy aging. IEEE Transactions on Information Technology in Biomedicine. 2004; 8(3):235-237. DOI: 10.1109/TITB. 2004.835531 [PubMed: 15484427]

10. Bewernitz MW, Mann WC, Dasler P, Belchior P. Feasibility of machine-based prompting to assist persons with dementia. Assistive Technology. 2009; 21(4):196-207. DOI: 10.1080/10400430903246050 [PubMed: 20066886]

11. Epstein JN, Willis MG, Conners CK, Johnson DE. Use of a technological prompting device to aid a student with attention deficit hyperactivity disorder to initiate and complete daily tasks: An exploratory study. Journal of Special Education Technology. 2001; 16(1):19-28.

12. Kaushik P, Intille SS, Larson K. User-adaptive Reminders for Home-based Medical Tasks. Methods of Information in Medicine. 2008; 47(3):203-207. [PubMed: 18473085]

13. Lamming, M., Flynn, M. Forget-me-not: Intimate computing in support of human memory. Proceedings FRIEND21, International Symposium on Next Generation Human Interface; 1994; p. 4

14. Tsui, KM., Yanco, HA. Prompting devices: a survey of memory aids for task sequencing. QoLT International Symposium: Intelligent Systems for Better Living; 2010;

15. Das, B., Seelye, AM., Thomas, BL., Cook, DJ., Holder, LB., Schmitter-Edgecombe, M. Using smart phones for context-aware prompting in smart environments. IEEE In Consumer Communications and Networking Conference; Las Vegas: IEEE; 2012. p. 399-403.

16. Seelye A, Schmitter-Edgecombe M, Das B, Cook D. Application of cognitive rehabilitation theory to the development of smart prompting technologies. IEEE Reviews in Biomedical Engineering. 2012; 5:29-44. DOI: 10.1109/RBME.2012.2196691 [PubMed: 23231987]

17. Robertson K, Rosasco C, Feuz K, Schmitter-Edgecombe M, Cook D. Prompting technologies: A comparison of time-based and context-aware transition-based prompting. Technology and Health Care. 2015; 23(6):745-756. DOI: 10.3233/THC-151033 [PubMed: 26409520]

18. Mihailidis A, Boger JN, Craig T, Hoey J. The COACH prompting system to assist older adults with dementia through handwashing: An efficacy study. BMC geriatrics. 2008; 8(1):28.doi: 10.1186/1471-2318-8-28 [PubMed: 18992135]

19. Pollack, ME., McCarthy, CE., Ramakrishnan, S., Tsamardinos, I., Brown, LE., Carrion, S., Colbry, D., Orosz, C., Peintner, B. Autominder: A planning, monitoring, and reminding assistive agent. 7th International Conference on Intelligent Autonomous Systems; 2002 (March); p. 265-272.

20. Boger J, Mihailidis A. The future of intelligent assistive technologies for cognition: devices under development to support independent living and aging-with-choice. NeuroRehabilitation. 2011; 28(3):271-280. DOI: 10.3233/NRE-2011-0655 [PubMed: 21558632]

21. Furniss F, Lancioni G, Rocha N, Cunha B, Seedhouse P, Morato P, O'Reilly MF. Vicaid: Development and evaluation of a palmtop-based job aid for workers with severe developmental disabilities. British Journal of Educational Technology. 2001; 32(3):277-287. DOI: 10.1111/1467-8535.00198

22. O’Neill B, Gillespie A. Simulating naturalistic instruction: the case for a voice mediated interface for assistive technology for cognition. Journal of Assistive Technologies. 2008; 2(2):22-31. DOI: 10.1108/17549450200800015

23. Laarhoven T, van Johnson J, Laarhoven-Myers T, van Grider K, Grider K. The effectiveness of using a video ipod as a prompting device in employment settings. Journal of Behavioral Education. 2009; 18(2):119-141.

24. Carmien, SP. Socio-technical environments supporting distributed cognition for persons with cognitive disabilities [dissertation]. Boulder: University of Colorado; 2006.

25. Wade TK, Troy JC. Mobile phones as a new memory aid: a preliminary investigation using case studies. Brain Injury. 2001; 15(4):305-320. DOI: 10.1080/026990501750111256 [PubMed: 11299132]

26. Clyburn LD, Stones MJ, Hadjistavropoulos T, Tuokko H. Predicting caregiver burden and depression in Alzheimer's disease. Journals of Gerontology Series B. 2000; 55(1):S2-13. DOI: 10.1093/geronb/55.1.S2 
27. Foster ER. Instrumental Activities of Daily Living Performance among People with Parkinson's disease Without Dementia. The American Journal of Occupational Therapy. 2014; 68(3):353-362. DOI: 10.5014/ajot.2014.010330 [PubMed: 24797199]

28. Bandura A. Self-efficacy: toward a unifying theory of behavioral change. Psychological Review. 1977; 84(2):191-215. DOI: 10.1037/0033-295X.84.2.191 [PubMed: 847061]

29. Bandura A. Social cognitive theory of self-regulation. Organizational Behavior and Human Decision Processes. 1991; 50(2):248-287. DOI: 10.1016/0749-5978(91)90022-L

30. De Frias CM, Dixon RA, Bäckman L. Use of memory compensation strategies is related to psychosocial and health indicators. The Journals of Gerontology Series B: Psychological Sciences and Social Sciences. 2003; 58(1):12-22. DOI: 10.1093/geronb/58.1.P12

31. Simon CM, Schmitter-Edgecombe M. The role of cognitive reserve and memory self-efficacy on compensatory strategy use: a structural equation approach. Journal of Clinical and Experimental Neuropsychology. 2016 (In press).

32. Bäckman L, Dixon RA. Psychological compensation: a theoretical framework. Psychological Bulletin. 1992; 112(2):259-283. DOI: 10.1037/0033-2909.112.2.259 [PubMed: 1454895]

33. Hertzog, C., Dixon, RA. Metacognitive development in adulthood and old age. Cambridge: MIT press; 1994.

34. Turvey CL, Schultz S, Arndt S, Wallace RB, Herzog R. Memory complaint in a community sample aged 70 and older. Journal of the American Geriatrics Society. 2000; 48(11):1435-1441. DOI: 10.1111/j.1532-5415.2000.tb02634 [PubMed: 11083320]

35. Brandt, J., Folstein, M. Telephone Interview for Cognitive Status. Lutz: Psychological Assessment Resources; 2003.

36. McKhann G, Drachman D, Folstein M, Katzman R, Price D, Stadlan EM. Clinical diagnosis of Alzheimer's disease report of the NINCDS-ADRDA work group under the auspices of Department of Health and Human Services Task Force on Alzheimer's Disease. Neurology. 1984; 34(7): 939.doi: 10.1212/WNL.34.7.939 [PubMed: 6610841]

37. Albert MS, DeKosky ST, Dickson D, Dubois B, Feldman HH, Fox NC, Gamst A, Holtzman DM, Jagust WJ, Petersen RC, Snyder PJ, Carrillo MC, Thies B, Phelps CH. The diagnosis of mild cognitive impairment due to Alzheimer's disease: Recommendations from the National Institute on Aging- Alzheimer's Association workgroups on diagnostic guidelines for Alzheimer's disease. Alzheimer's \& Dementia. 2011; 7(3):270-279. DOI: 10.1016/j.jalz.2011.03.008

38. PROMIS, P. Patient Reported Outcomes Measurement Information System. National Health Institute; 2012.

39. Williams, JM. MAS: Memory assessment scales: Professional manual. Psychological Assessment Resources; 1991.

40. Delis, DC., Kaplan, E., Kramer, JH. Delis-Kaplan executive function system (D-KEFS). Psychological Corporation; 2001.

41. MacDonald PL, Gardner RC. Type I error rate comparisons of post hoc procedures for I j ChiSquare tables. Educational and Psychological Measurement. 2000; 60(5):735-754. DOI: $10.1177 / 00131640021970871$

42. Sharpe D. Your Chi-Square Test is Statistically Significant: Now What? Practical Assessment, Research \& Evaluation. 2015; 20(8) http://pareonline.net/getvn.asp?v=20\&n=8 retrieved April 15, 2016. 


\section{Table 1}

Frequencies of participant diagnoses $(n=170)$;

\begin{tabular}{lcccc}
\hline Parameter & Raw & $\%$ & CC & FCC \\
\hline Healthy older adults & 114 & 67.1 & 41 & 73 \\
Mild cognitive impairment & 20 & 11.8 & 17 & 3 \\
Parkinson's disease & 12 & 7.1 & 6 & 6 \\
Stroke & 6 & 3.5 & 6 & 0 \\
Traumatic brain injury & 5 & 2.9 & 3 & 2 \\
Other medical conditions (e.g. cancer) & 13 & 7.6 & 12 & 1 \\
\hline
\end{tabular}

FCC=Few Cognitive Complaints; $\mathrm{CC}=$ Cognitive Complaints 
Table 2

Demographics and cognitive measures by cognitive complaints groups;

\begin{tabular}{lrrrc}
\hline \multirow{2}{*}{ Parameter } & \multicolumn{2}{c}{ Mean \pm Standard deviation } & & T \\
\cline { 2 - 5 } & $66.76 \pm 8.92$ & $67.24 \pm 8.55$ & -0.351 & 0.056 \\
\hline Age & $16.38 \pm 2.36$ & $16.55 \pm 2.38$ & -0.486 & 0.072 \\
Education & $7.73 \pm 2.49^{a}$ & $9.15 \pm 2.62^{a}$ & $-3.58^{*}$ & 0.556 \\
Immediate prose memory & FCC & & \\
Delayed prose memory & $7.44 \pm 2.74^{a}$ & $8.87 \pm 2.50^{a}$ & $-3.53^{*}$ & 0.545 \\
Verbal fluency & $11.84 \pm 3.54$ & $13.04 \pm 3.41$ & $-2.25^{*}$ & 0.345 \\
Design fluency & $7.17 \pm 2.58^{a}$ & $7.41 \pm 2.26$ & -0.643 & 0.099 \\
\hline
\end{tabular}

$\mathrm{CC}=$ Cognitive Complaints $(\mathrm{n}=85)$; $\mathrm{FCC}=\mathrm{Few}$ Cognitive Complaints $(\mathrm{n}=85)$;

${ }_{\mathrm{n}}^{a}<85$;

p $<0.05$ 


\section{Table 3}

Preferred prompt level percentages

\begin{tabular}{lccc}
\multirow{2}{*}{ Complaints group } & \multicolumn{3}{c}{ Prompt level preferred, \% } \\
\cline { 2 - 4 } & Indirect & Direct & Multimodal \\
\hline Cognitive Complaints & 15.3 & 35.3 & 49.4 \\
Few Cognitive Complaints & 25.9 & 44.7 & 29.4 \\
\hline
\end{tabular}




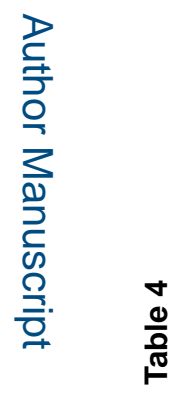

产

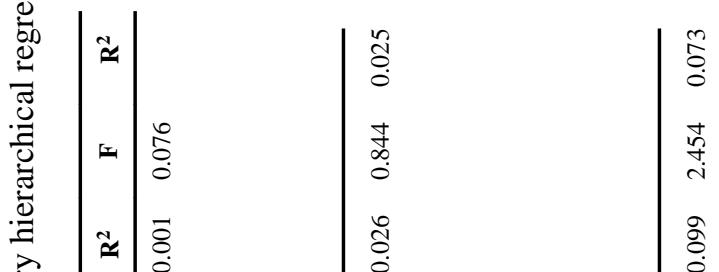

空

文

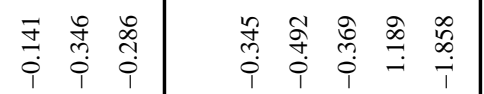

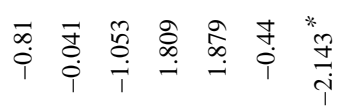

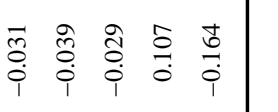

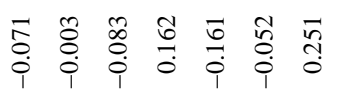

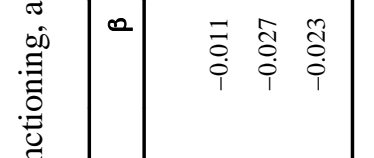

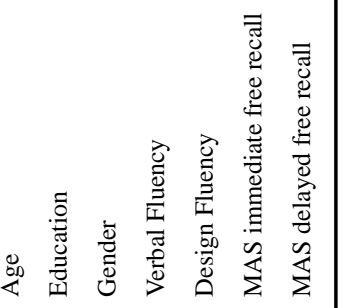

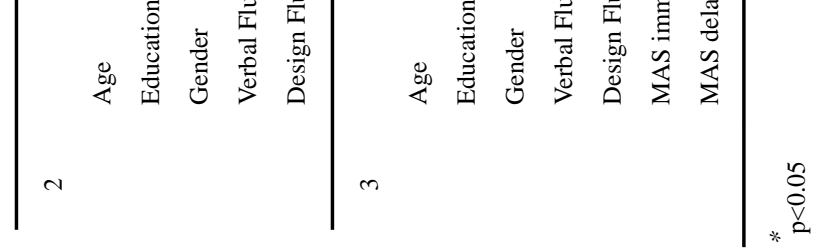

Gerontechnology. Author manuscript; available in PMC 2017 September 13. 